\title{
Bladder Recurrence Following the Treatment of Upper Tract Urothelial Carcinoma
}

Takarn Itsaranujareankul, M.D., Kanrapee Nuwatkrisin, M.D., Kamol Panumatrassamee, M.D., Dutsadee Sowanthip, M.D., Julin Opanuraks, M.D., Supoj Ratchanon, M.D., Apirak Santingamkun, M.D., Manint Usawachintachit, M.D.

Division of Urology, Department of Surgery, Faculty of Medicine, King Chulalongkorn Memorial Hospital, Chulalongkorn University, The Thai Red Cross Society, Patumwan, Bangkok 10330, Thailand.

Received 15 December 2020 • Revised 3 February 2021 • Accepted 8 February 2021 • Published online 30 April 2021

\begin{abstract}
:
Objective: Although, upper tract urothelial carcinoma (UTUC) is rare it is associated with a high mortality rate and frequently, followed by bladder recurrence after radical surgery. Hence, this study aimed to identify the rate of bladder recurrence and its predictive factors.
\end{abstract}

Material and Methods: We reviewed the medical records of 104 patients, who were diagnosed with UTUC and had radical nephroureterectomy (RNU), at the King Chulalongkorn Memorial Hospital. We excluded patients who have concurrent bladder cancer, or had a history of bladder cancer. Various clinicopathological factors were analyzed using the log-rank test and Cox proportional hazard model.

Results: The mean age at diagnosis of UTUC was 68 years, and one-third of the patients were diagnosed with pathological T3 (33.7\%). The mean follow-up duration was 56 months. Bladder recurrence occurred in 39 out of 104 patients $(37.5 \%)$, and the median time to recurrence was 5.8 months (interquartile range 3.6 to 11.0 months). Tumor location in the distal ureter $(p-v a l u e=0.038)$ and history of diagnostic ureteroscopy $(p-v a l u e=0.004)$ were significantly associated with bladder recurrence in the univariate model. However, only the history of diagnostic ureteroscopy remained significant in the multivariate analysis ( $p$-value $=0.023$ ).

Contact: Manint Usawachintachit, M.D.

Division of Urology, Department of Surgery, Faculty of Medicine,

King Chulalongkorn Memorial Hospital, Chulalongkorn University,

The Thai Red Cross Society, Patumwan, Bangkok 10330, Thailand

E-mail: manint.u@chula.ac.th

๑) 2021 JHSMR. Hosting by Prince of Songkla University. All rights reserved.

This is an open access article under the CC BY-NC-ND license

(http://www.jhsmr.org/index.php/jhsmr/about/editorialPolicies\#openAccessPolicy).
J Health Sci Med Res 2022;40(1):1-9 doi: 10.31584 /jhsmr.2021807 www.jhsmr.org 
Conclusion: Bladder recurrence, following RNU, occurs in one-third of patients. Potential predictive factors may include history of diagnostic ureteroscopy, and the tumor location being in the distal ureter.

Keywords: bladder recurrence, nephroureterectomy, upper tract urothelial carcinoma

\section{Introduction}

Upper tract urothelial carcinoma (UTUC) accounts for $5.0-10.0 \%$ of all urothelial carcinoma cases, with an incidence of two cases per 100,000 population, per year. ${ }^{1}$ The standard treatment of UTUC is radical nephroureterectomy (RNU) with bladder cuff excision. ${ }^{1}$

Following radical surgery, recurrent urothelial carcinoma in the urinary bladder may occur in up to $50.0 \%$ of patients. $^{2}$ Possible explanations for this include: inappropriate surgical manipulation during RNU, the inadequacy of distal ureterectomy, and the theoretical field change of urothelial carcinoma in the entire urinary tract. Previous risk factors reported to predict bladder recurrence are tumor multifocality ${ }^{3-6}$, carcinoma in situ (CIS) in the upper urinary tract ${ }^{3,7}$, tumor grade ${ }^{8,9}$, tumor stage ${ }^{5,6,10}$, tumor location $^{10-12}$, and gender. ${ }^{9}$ However, there are discordant findings from previous studies. For example, while one group found that diagnostic ureteroscopy is predictive for bladder recurrence ${ }^{13}$, others demonstrated no association. ${ }^{14,15}$ Moreover, some studies included patients with prior history of bladder cancer, which may have different pathogenesis of bladder recurrence. ${ }^{2,3,10}$ Therefore, we proposed this study to find the incidence rate of de novo bladder recurrence, following RNU, and its potential predictive factors.

\section{Material and Methods}

This study was a retrospective review of adult patients diagnosed with UTUC, and who were treated at the King Chulalongkorn Memorial Hospital, Bangkok, Thailand; from January 2004 to December 2018. The institutional review board approved the study (IRB\# 496/62). Informed consent was waived. We included all localized or locally advanced UTUC patients, over 18 years of age, who underwent RNU at our institution. We excluded patients who had a short duration of postoperative follow-up periods (less than six months), previous history of bladder cancer, or concurrent bladder cancer at the time of UTUC diagnosis. Patient characteristics such as age, gender, disease laterality, comorbidity, and presenting symptoms were collected. Tumor characteristics (size and location) were defined as based on the postoperative pathological reports. Tumor size was measured at the maximal dimension and divided into two groups: < four $\mathrm{cm}$ and $\geq$ four $\mathrm{cm}$. Tumor location was categorized into five groups: renal calyx, renal pelvis, proximal ureter, mid ureter, and distal ureter. Multifocal tumor was defined as tumors located in $\geq$ two areas. Some patients had diagnostic ureteroscopy prior to radical surgery, due to an inconclusive clinical diagnosis. However, none of the patients in this study received ureteroscopic tumor ablation. Some patients received a ureteral stent placement; due to renal insufficiency, intractable pain, or secondary infection. Delay in surgery was marked if RNU was performed later than 90 days from the initial presentation. The surgical approach for RNU procedures (open or laparoscopic) was based on the surgeon's discretion. However, all distal ureterectomy and bladder cuff excision procedures were performed via the open extravesical technique. Adjuvant intravesical or upper tract instillation, with either chemotherapy or BCG, was not utilized during the study period. For pathological features, we relied on histopathology derived at the time of RNU. Tumor staging and grading were reported using the $8^{\text {th }}$ edition of the American Joint Committee on Cancer TMN Staging System for renal pelvis and ureter. ${ }^{16}$ 
The study endpoint was the presence of urothelial carcinoma in the urinary bladder, also known as bladder recurrence. Following RNU, all patients underwent bladder surveillance; routine office-based cystoscopy was performed every three months, for the first two years; then every six months, for the next two years; and annually thereafter. Abnormal voiding symptoms, such as hematuria or dysuria, also led to immediate cystoscopy. Suspicious lesions would be pathologically proven. We also performed contrastenhanced imaging to evaluate local recurrence or distant metastasis yearly.

Continuous variables were reported as mean \pm standard deviation, with categorical variables being presented as frequency and percentage. The predictive value of clinicopathological factors was analyzed in relation to the elapsed time to bladder recurrence. The survival curves were obtained using the Kaplan-Meier method. Univariate analysis was used to obtain predictive factors of bladder recurrence. Subsequent multivariate analysis was performed with the Cox proportional hazard model. Statistical analysis was performed using IBM SPSS Statistics version 22.0 (SPSS Inc., Chicago, IL, USA). A $\mathrm{p}$-value $<0.050$ was considered to be statistically significant.

\section{Results}

The medical records of 186 , localized or locally advanced, UTUC patients were reviewed during the study period. Sixty patients were excluded, due to previous history of bladder cancer or concomitant diagnosis of bladder cancer. Seventeen patients were excluded because they had a short duration of postoperative follow-up periods, and five patients underwent nephron-sparing or endoscopic surgery instead of RNU. Finally, 104 patients were included in the analysis.

The mean age at UTUC diagnosis was 68 years, and $56.7 \%$ were females. Hypertension (61.5\%) and diabetes mellitus (26.0\%) were found in a significant number of patients. The most common clinical presentation was gross hematuria $(79.8 \%)$, followed by flank pain (12.5\%). Almost half of the patients had UTUC in the renal pelvis $(48.1 \%)$, and multifocal tumors were found in $38.5 \%$ of patients. Thirty-five from 104 patients (33.7\%) underwent ureteroscopy for UTUC diagnosis, and 14 patients (13.5\%) received ureteral stent placement prior to radical surgery (Table 1).

Table 1 Demographics and tumor characteristics of upper tract urothelial carcinoma patients

\begin{tabular}{ll}
\hline Parameters (n=104) & Value \\
\hline Age, mean \pm S.D. (years) & $68.0 \pm 11.9$ \\
Sex, $\mathrm{n}(\%)$ & \\
$\quad$ Male & $45(43.3)$ \\
Female & $59(56.7)$ \\
Comorbidity, $\mathrm{n}(\%)$ & \\
$\quad$ Diabetes mellitus & $27(26.0)$ \\
Hypertension & $64(61.5)$ \\
Dyslipidemia & $24(23.1)$ \\
Ischemic heart disease & $6(5.8)$ \\
Stroke/CVA & $8(7.7)$ \\
COPD & $2(1.9)$ \\
Clinical presentation, $\mathrm{n}(\%)$ & \\
$\quad$ Asymptomatic & $11(10.6)$ \\
Gross hematuria & $83(79.8)$ \\
Microscopic hematuria & $2(1.9)$ \\
Flank pain & $13(12.5)$ \\
Palpable abdominal mass & $1(1.0)$ \\
Laterality, $\mathrm{n}$ (\%) & \\
Right & $53(51.0)$ \\
Left & $47(45.2)$ \\
Bilateral & $4(3.8)$ \\
Tumor location, $\mathrm{n}$ (\%) & \\
Renal calix & $43(41.3)$ \\
Renal pelvis & $50(48.1)$ \\
Proximal ureter & $26(25.0)$ \\
Mid ureter & $13(12.5)$ \\
Distal ureter & $33(31.7)$ \\
Multifocal tumor, $\mathrm{n}$ (\%) & $40(38.5)$ \\
Diagnostic ureteroscopy prior to radical surgery, & $35(33.7)$ \\
$\mathrm{n}$ (\%) & \\
Ureteral stent placement prior to radical surgery, & $14(13.5)$ \\
$\mathrm{n}$ (\%) & \\
\hline & \\
\hline & \\
\hline
\end{tabular}

S.D.=standard deviation, CVA=cerebrovascular accident, COPD=chronic obstructive lung disease 
Mean duration from UTUC presentation to RNU was 112.4 days. Surgery was delayed (>90 days) in 46 patients (44.2\%). Fifty four percent of patients had open RNU, whilst $46.2 \%$ had laparoscopy. As to the histopathological results, one-third of the patients had pathological stage T3 (33.7\%), followed by pathological stage T1 (27.9\%) and pathological stage Ta (18.3\%). High-grade tumors were detected in $84.6 \%$ of patients; whereas, CIS was only found in $8.7 \%$ of patients (Table 2).

Table 2 Perioperative data and pathological characteristics of upper tract urothelial carcinoma

\begin{tabular}{ll}
\hline Parameters $(\mathrm{n}=104)$ & Value \\
\hline $\begin{array}{l}\text { Duration from UTUC diagnosis to surgery, } \\
\text { mean } \pm \text { S.D. (days) }\end{array}$ & $112.4 \pm 109.8$ \\
Delay in surgery (>90 days), $\mathrm{n}(\%)$ & $46(44.2)$ \\
Surgical approach, $\mathrm{n}(\%)$ & \\
$\quad$ Open & $56(53.8)$ \\
Laparoscopic & $48(46.2)$ \\
Pathological T staging, $\mathrm{n}(\%)$ & $19(18.3)$ \\
pTa & $29(27.9)$ \\
pT1 & $17(16.3)$ \\
pT2 & $35(33.7)$ \\
pT3 & $4(3.8)$ \\
pT4 & \\
Tumor grade, $n(\%)$ & $16(15.4)$ \\
Low grade & $88(84.6)$ \\
High grade & $9(8.7)$ \\
Presence of CIS, $n(\%)$ & \\
\hline
\end{tabular}

UTUC=upper tract urothelial carcinoma, S.D.=standard deviation, $\mathrm{CIS}=$ carcinoma in situ

Postoperatively, the mean follow-up duration was 56 months (ranging from 6 to 184 months). During the study period, 39 patients $(37.5 \%)$ had bladder recurrence. The median time interval from surgery to bladder recurrence was 5.8 months. All recurrences had transurethral resection, and we detected the same tumor grade as in the upper tract in $28 / 36$ patients $(77.8 \%)$. The bladder pathology was unavailable in three patients, as these received endoscopic resection elsewhere (Table 3 ).

The Kaplan-Meier method demonstrated that: prior history of diagnostic ureteroscopy $(p-v a l u e=0.004)$ and the tumor location in the distal ureter $(p-v a l u e=0.038)$ were significantly associated with bladder recurrence (Figure 1). The delay in radical surgery had a considerable association, but did not reach a statistically significant level $(p-v a l u e=$ 0.054). In addition, the pathological stage, tumor grade, multifocality, size, concomitant CIS in the upper tract, and prior ureteral stent placement did not predict subsequent bladder recurrence. From the multivariate analysis, only history of diagnostic ureteroscopy remained as significantly associated with developing bladder cancer following RNU [hazard ratio $(\mathrm{HR})=2.15 ; 95 \%$ confidence interval $(\mathrm{Cl}) 1.11$ to $4.14, p$-value=0.023] (Table 4).

Table 3 Characteristics of bladder recurrence

\begin{tabular}{ll}
\hline Parameters $(\mathbf{n = 3 9 )}$ & Value \\
\hline $\begin{array}{l}\text { Duration from radical nephroureterectomy to } \\
\text { bladder recurrence, median (IQR) (months) }\end{array}$ & $5.8(3.6-11.0)$ \\
Pathological T staging, $\mathrm{n}(\%)^{*}$ & \\
CIS & $2(5.6)$ \\
pTa & $15(41.7)$ \\
pT1 & $17(47.2)$ \\
pT2 & $1(2.8)$ \\
pT3 & $1(2.8)$ \\
Tumor grade, $\mathrm{n}(\%)^{*}$ & $10(27.8)$ \\
Low grade & $26(72.2)$ \\
High grade & $28(77.8)$ \\
Presence of the same tumor grade as in the & \\
upper urinary tract, $\mathrm{n}(\%)^{*}$ & \\
\hline
\end{tabular}

*Data was available in 36/39 patients

$\mathrm{IQR}=$ interquartile range, $\mathrm{CIS}=$ carcinoma in situ 


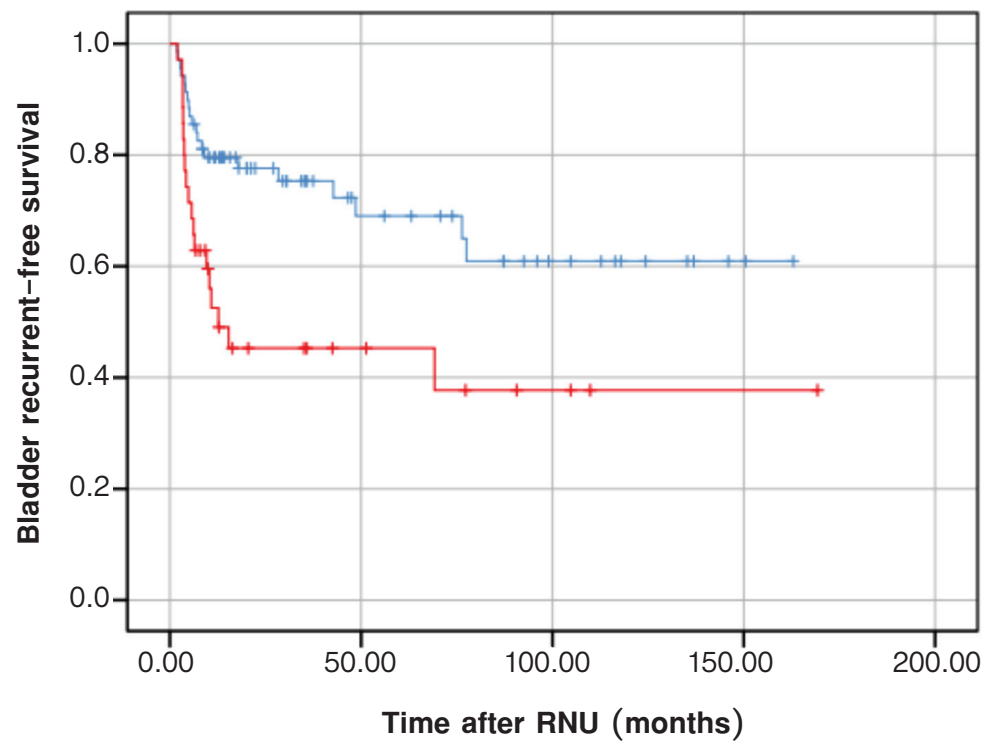

\section{Diagnosis \\ ureteroscopy \\ $\neg$ No \\ + No-censored \\ + Yes-censored}

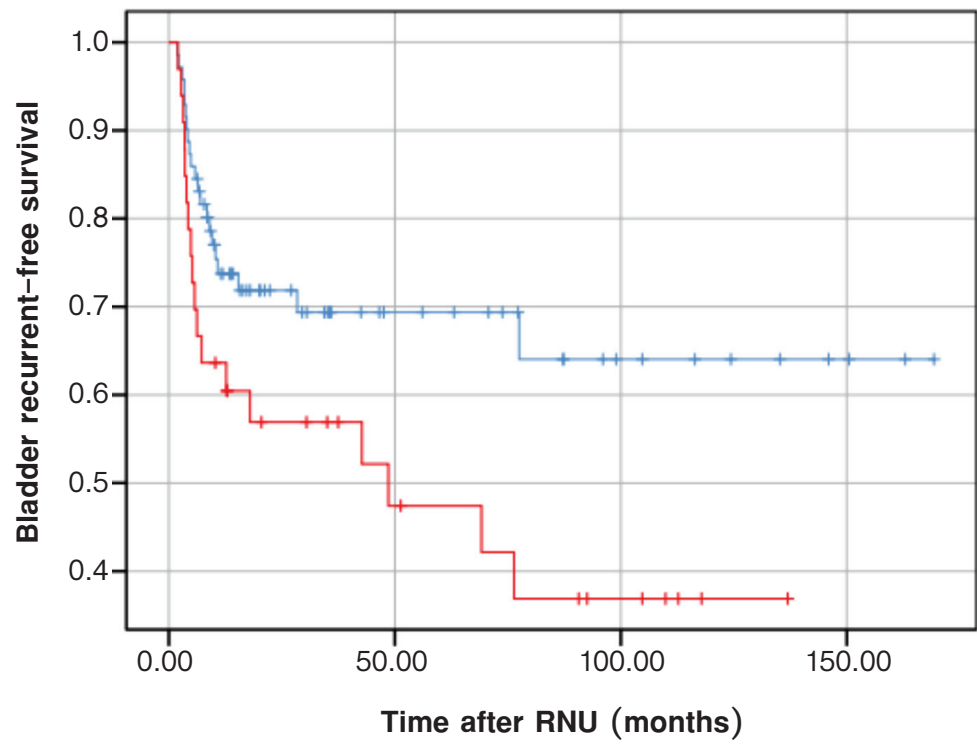

Tumor location in the distal ureter

$\neg$ No

$\neg$ Yes

+ No-censored

+ Yes-censored

$\mathrm{RNU}=$ radical nephroureterectomy

Figure 1 Kaplan-Meier plot of bladder recurrence-free survival comparing patients with previous history of diagnostic ureteroscopy $(p-$ value $=0.004)$ and tumor location in the distal ureter $(p-$ value $=0.038)$ 
Table 4 Clinicopathological risk factors of bladder recurrence

\begin{tabular}{|c|c|c|c|}
\hline \multicolumn{4}{|c|}{ Univariate analysis } \\
\hline \multicolumn{2}{|c|}{ Parameters $(n=104)$} & \multicolumn{2}{|l|}{$\mathrm{p}$-value } \\
\hline \multicolumn{2}{|c|}{ Age ( $<70$ vs $\geq 70$ years) } & \multicolumn{2}{|l|}{0.177} \\
\hline \multicolumn{2}{|c|}{ Sex } & \multicolumn{2}{|l|}{0.523} \\
\hline \multirow[t]{3}{*}{ Comorbidity } & Hypertension & \multicolumn{2}{|l|}{0.872} \\
\hline & Diabetes mellitus & \multicolumn{2}{|l|}{0.915} \\
\hline & Dyslipidemia & \multicolumn{2}{|l|}{0.951} \\
\hline \multicolumn{2}{|c|}{ History of gross hematuria at presentation } & \multicolumn{2}{|l|}{0.523} \\
\hline \multirow[t]{5}{*}{ Tumor location } & Renal calyx & \multicolumn{2}{|l|}{0.355} \\
\hline & Renal pelvis & \multicolumn{2}{|l|}{0.987} \\
\hline & Proximal ureter & \multicolumn{2}{|l|}{0.524} \\
\hline & Mid ureter & \multicolumn{2}{|l|}{0.291} \\
\hline & Distal ureter & \multicolumn{2}{|l|}{$0.038^{*}$} \\
\hline \multicolumn{2}{|c|}{ Tumor multifocality } & \multicolumn{2}{|l|}{0.188} \\
\hline \multicolumn{2}{|c|}{ Tumor size ( $<4$ vs $\geq 4 \mathrm{~cm})$} & \multicolumn{2}{|l|}{0.791} \\
\hline \multicolumn{2}{|c|}{ Ureteroscopy prior to radical surgery } & \multicolumn{2}{|l|}{$0.004^{*}$} \\
\hline \multicolumn{2}{|c|}{ Ureteral stent placement prior to radical surgery } & 0.675 & \\
\hline Delay in radical & urgery ( $\geq 90$ days from the initial presentation) & 0.054 & \\
\hline Surgical approa & (Laparoscopic vs open) & 0.186 & \\
\hline Bladder cuff exc & ion & 0.936 & \\
\hline Pathological T s & ge (pTa and pT1 vs pT2-4) & 0.456 & \\
\hline Tumor grade (lo & grade vs high grade) & 0.850 & \\
\hline Concomitant $\mathrm{ClS}$ & in the upper urinary tract & 0.776 & \\
\hline & Multiv & & \\
\hline Parameters $(n=$ & 05) & HR & $p$-value \\
\hline Tumor location: & istal ureter & 1.57 (0.81 to 3.03$)$ & 0.179 \\
\hline Ureteroscopy pr & $r$ to radical surgery & 2.15 (1.11 to 4.14$)$ & $0.023^{*}$ \\
\hline
\end{tabular}

$\mathrm{CIS}=$ carcinoma in situ, $\mathrm{HR}=$ hazard ratio

\section{Discussion}

Several hypotheses may explain the etiology of bladder recurrence following RNU. During the operation, downstream cancer cells shedding from surgical manipulation could cause tumor implantation in the urinary bladder. Inadequacy of distal ureterectomy, or bladder cuff excision from a limited working space may leave some tumor cells in the surgical bed. ${ }^{9}$ Furthermore, urothelial carcinoma is a potential "field-change" disease, with the entire urothelium at risk of malignant transformation. ${ }^{2}$
In our UTUC cohort, from those who received RNU, the bladder recurrence rate was $37.5 \%$. This incidence was comparable to previous reports $(15.0-50.0 \%)^{2-12}$; wherein, if the postoperative follow-up period was longer, then the recurrence rate would be higher. This finding underscores the need for rigorous cystoscopy surveillance at regular intervals. The median time-lapse from RNU to bladder recurrence was 5.8 months, which is considered short when compared to previous reports (range 6.9-13.2 months). ${ }^{5,11,12}$ 
In our study, the most relevant predictive factor for bladder recurrence was previous history of diagnostic ureteroscopy. With the development of endoscopic devices, ureteroscopy has become a useful tool in accurately diagnosing UTUC. However, concerns have been raised that ureteroscopic manipulation and tissue biopsy may result in tumor implantation. ${ }^{17}$ Increased intraluminal pressure and retrograde flow during the procedure may lead to the shedding of tumor cells, which then implant at the bladder urothelial surface. ${ }^{17}$ Currently, there is growing evidence that ureteroscopy was positively correlated with bladder recurrence after RNU. Lee et al., retrospectively divided UTUC patients according to the timing of diagnostic ureteroscopy: no ureteroscopy group, 1-session group (diagnostic ureteroscopy immediately followed by RNU), and 2-session group (delayed RNU after diagnostic ureteroscopy). The incidence of bladder recurrence was significantly higher in the 2-session group compared to the no URS group (HR=3.82; 95\% Cl 1.44 to 10.13). ${ }^{18}$

Aside from this, in the univariate model our findings corroborated previous reports that tumor location in the distal ureter may increase the risk of bladder recurrence. 8,11,12 However, in the multivariate model this was not significantly associated with bladder recurrence. The distal tumor location could ease tumor cell spillage into the bladder. Moreover, higher urine flow rate and intraluminal pressure in the ureter may lead to increased tumor cell detachment. ${ }^{8}$ Some authors suggested that early intravesical chemotherapy might be beneficial in preventing bladder recurrence in patients who had UTUC in the distal ureter. ${ }^{8}$ Nevertheless, this suggestion has yet to be confirmed by a randomized trial.

We could not demonstrate a significant association between the delay in RNU and bladder recurrence. Our finding is similar to the previous report from Raman et al., who used a cut-point of 1.5 months for the definition of delayed RNU. ${ }^{2}$ UTUC is an aggressive tumor that rapidly progresses and metastasizes. Hence, RNU is a time- sensitive procedure, and the delay in RNU may result in the up-staging of the primary tumor. ${ }^{19}$ In contrast, the etiology of bladder recurrence may be an accumulative and slow process. Therefore, the delay of surgery by a matter of a few months is unlikely to impact the risk of bladder recurrence.

Employing various measures may reduce bladder recurrence after RNU. Intraoperatively, general advice is to clip or ligate the ureter distal to the tumor site. This practice is likely to prevent tumor cells from spreading along the ureter during mechanical manipulation. ${ }^{8}$ Nevertheless, data to support this practice are still lacking. Prophylactic intravesical chemotherapy instillation following RNU appears to reduce the risk. ${ }^{20,21}$ However, the optimal regimen and the time of instillation are still debatable. O'Brien et al., prospectively compared an immediate single dose of mitomycin-C (40 mg) to standard of care. They found that there was an $11.0 \%$ absolute risk reduction of bladder recurrence within the first year following RNU. ${ }^{22}$ Likewise, Ito et al., compared a single dose of pirarubicin (30 mg within 48 hours) to no instillation in their randomized trial, and discovered that there was a decreased risk of bladder recurrence in the pirarubicin group $(\mathrm{HR}=0.26$; $95 \% \mathrm{Cl} 0.07$ to 0.91$).{ }^{21}$ Furthermore, the European Association of Urology Guidelines on Upper Urinary Tract Urothelial Carcinoma recommends postoperative bladder instillation following RNU to lower the bladder recurrence rate. ${ }^{1}$ In addition, patients with risk factors should periodically have a cystoscopy.

There are some limitations in this study. First, its retrospective design may have led to selection bias. We excluded patients who had concurrent bladder cancer or history of bladder cancer. However, some patients could have already had an invisible or very small bladder tumor, and were included in this study. Second, there were multiple surgeons who performed the operations, and this may have caused bias, because they used different surgical techniques, and have various levels of experience. Each surgeon independently decided whether 
to perform diagnostic ureteroscopy or selected the surgical approach, without any strict criteria. Some surgeons utilized postoperative urine cytology for bladder surveillance, while some did not. Third, the data from this study was obtained from a single tertiary-care center, which may not be applicable to other hospitals. Fourth, the sample size in this study was relatively small, because UTUC is rare. Statistically, the dataset might not be powerful enough to detect a small difference among the different categories of patients. Last, we did not include perioperative systemic chemotherapy or radiotherapy in the analysis, due to a considerable variation in the indications, regimens, and number of cycles.

\section{Conclusion}

Bladder recurrence following RNU occurs in $37.5 \%$ of patients, and the median time interval from surgery to bladder recurrence was 5.8 months. The potential predictive factors may include history of diagnostic ureteroscopy, and the tumor location in the distal ureter. However, only history of diagnostic ureteroscopy remains statistically significant in the multi-variate analysis. A large-scale, multi-institutional study; with a prospective design should be employed to verify this finding.

\section{Funding sources}

None

\section{Conflict of interest}

None

\section{References}

1. Roupret M, Babjuk M, Comperat E, Zigeuner R, Sylvester RJ, Burger M, et al. European Association of Urology Guidelines on Upper Urinary Tract Urothelial Carcinoma: 2017 Update. Eur Urol 2018;73:111-22.

2. Raman JD, Ng CK, Boorjian SA, Vaughan ED Jr, Sosa RE, Scherr DS. Bladder cancer after managing upper urinary tract transitional cell carcinoma: predictive factors and pathology. BJU Int 2005;96:1031-5.

3. Xylinas E, Colin P, Audenet F, Phe V, Cormier L, Cussenot O, et al. Intravesical recurrence after radical nephroureterectomy for upper tract urothelial carcinomas: predictors and impact on subsequent oncological outcomes from a national multicenter study. World J Urol 2013;31:61-8.

4. Terakawa T, Miyake H, Muramaki M, Takenaka A, Hara I, Fujisawa M. Risk factors for intravesical recurrence after surgical management of transitional cell carcinoma of the upper urinary tract. Urology 2008;71:123-7.

5. Hisataki T, Miyao N, Masumori N, Takahashi A, Sasai M, Yanase $\mathrm{M}$, et al. Risk factors for the development of bladder cancer after upper tract urothelial cancer. Urology 2000;55: 663-7.

6. Matsui Y, Utsunomiya N, Ichioka K, Ueda N, Yoshimura K, Terai $A$, et al. Risk factors for subsequent development of bladder cancer after primary transitional cell carcinoma of the upper urinary tract. Urology 2005;65:279-83.

7. Pieras E, Frontera G, Ruiz X, Vicens A, Ozonas M, Piza P. Concomitant carcinoma in situ and tumour size are prognostic factors for bladder recurrence after nephroureterectomy for upper tract transitional cell carcinoma. BJU Int 2010;106: 1319-23.

8. Zigeuner RE, Hutterer G, Chromecki T, Rehak P, Langner C. Bladder tumour development after urothelial carcinoma of the upper urinary tract is related to primary tumour location. BJU Int 2006;98:1181-6.

9. Huang WW, Huang HY, Liao AC, Shiue YL, Tai HL, Lin CM, et al. Primary urothelial carcinoma of the upper tract: important clinicopathological factors predicting bladder recurrence after surgical resection. Pathol Int 2009;59:642-9.

10. Novara G, De Marco V, Dalpiaz O, Gottardo F, Bouygues V, Galfano $A$, et al. Independent predictors of metachronous bladder transitional cell carcinoma (TCC) after nephroureterectomy for TCC of the upper urinary tract. BJU Int 2008; 101:1368-74.

11. Chung SD, Huang KH, Lai MK, Huang CY, Chen CH, Pu YS, et al. CKD as a risk factor for bladder recurrence after nephroureterectomy for upper urinary tract urothelial carcinoma. Am J Kidney Dis 2007;50:743-53.

12. Kobayashi $Y$, Saika T, Miyaji $Y$, Saegusa M, Arata R, Akebi N, et al. Preoperative positive urine cytology is a risk factor for 
subsequent development of bladder cancer after nephroureterectomy in patients with upper urinary tract urothelial carcinoma. World J Urol 2012;30:271-5.

13. Sung $H H$, Jeon $H G$, Han $D H$, Jeong $B C$, Seo SI, Lee HM, et al. Diagnostic Ureterorenoscopy Is Associated with Increased Intravesical Recurrence following Radical Nephroureterectomy in Upper Tract Urothelial Carcinoma. PloS One 2015;10: e0139976.

14. Lee HY, Yeh HC, Wu WJ, He JS, Huang CN, Ke HL, et al. The diagnostic ureteroscopy before radical nephroureterectomy in upper urinary tract urothelial carcinoma is not associated with higher intravesical recurrence. World $\mathrm{J}$ Surg Oncol 2018;16:135.

15. Ishikawa S, Abe T, Shinohara N, Harabayashi T, Sazawa A, Maruyama S, et al. Impact of diagnostic ureteroscopy on intravesical recurrence and survival in patients with urothelial carcinoma of the upper urinary tract. J Urol 2010;184:883-7.

16. Amin MB, Edge S, Greene F, Byrd DR, Brookland RK, Washington MK, et al. AJCC cancer staging manual. New York: Springer; 2017.

17. Liu P, Su XH, Xiong GY, Li XS, Zhou LQ. Diagnostic Ureteroscopy for Upper Tract Urothelial Carcinoma is Independently Associated with Intravesical Recurrence after Radical Nephroureterectomy. Int Braz J Urol 2016;42:1129-35.
18. Lee JK, Kim KB, Park YH, Oh JJ, Lee S, Jeong CW, et al. Correlation Between the Timing of Diagnostic Ureteroscopy and Intravesical Recurrence in Upper Tract Urothelial Cancer. Clin Genitourin Cancer 2016;14:e37-41.

19. Waldert M, Karakiewicz PI, Raman JD, Remzi M, Isbarn H, Lotan $\mathrm{Y}$, et al. A delay in radical nephroureterectomy can lead to upstaging. BJU Int 2010;105:812-7.

20. Wu WJ, Ke HL, Yang YH, Li CC, Chou YH, Huang CH. Should patients with primary upper urinary tract cancer receive prophylactic intravesical chemotherapy after nephroureterectomy?. J Urol 2010;183:56-61.

21. Ito A, Shintaku I, Satoh M, Ioritani N, Aizawa M, Tochigi T, et al. Prospective randomized phase II trial of a single early intravesical instillation of pirarubicin (THP) in the prevention of bladder recurrence after nephroureterectomy for upper urinary tract urothelial carcinoma: the THP Monotherapy Study Group Trial. J Clin Oncol 2013;31:1422-7.

22. O'Brien T, Ray E, Singh R, Coker B, Beard R, British Association of Urological Surgeons Section of Oncology. Prevention of bladder tumours after nephroureterectomy for primary upper urinary tract urothelial carcinoma: a prospective, multicentre, randomised clinical trial of a single postoperative intravesical dose of mitomycin C (the ODMIT-C Trial). Eur Urol 2011;60: 703-10. 